\title{
Disturbed neurovascular coupling in hemodialysis patients
}

\author{
Mei Jin ${ }^{1}$, Liyan Wang ${ }^{2}$, Hao Wang ${ }^{1}$, Xue Han ${ }^{2}$, Zongli Diao ${ }^{2}$, Wang Guo ${ }^{2}$, Zhenghan Yang ${ }^{1}$, Heyu Ding ${ }^{1}$, Zheng \\ Wang ${ }^{1}$, Peng Zhang ${ }^{1}$, Pengfei Zhao ${ }^{1}$, Han Lv $^{1}$, Wenhu Liu ${ }^{2}$, Zhenchang Wang ${ }^{\text {Corresp. } 1}$ \\ ${ }^{1}$ Department of Radiology, Beijing Friendship Hospital, Capital Medical University, Beijing, China \\ 2 Department of Nephrology, Faculty of Kidney Diseases, Beijing Friendship Hospital, Capital Medical University, Beijing, China \\ Corresponding Author: Zhenchang Wang \\ Email address: cjr.wzhch@vip.163.com
}

Background. Altered cerebral blood flow (CBF) and amplitude of low-frequency fluctuation (ALFF) have been reported in hemodialysis patients. However, neurovascular coupling impairments, which provide a novel insight into the human brain, have not been reported in hemodialysis patients. Methods. We combined arterial spin labeling (ASL) and blood oxygen level dependent (BOLD) techniques to investigate neurovascular coupling alterations and its relationships with demographic and clinical data in 46 hemodialysis patients and 47 healthy controls. To explore regional neuronal activity, ALFF was obtained from resting-state functional MRI. To measure cerebral vascular response, CBF was calculated from ASL. The across-voxel CBF-ALFF correlations for global neurovascular coupling and CBF/ALFF ratio for regional neurovascular coupling were compared between hemodialysis patients and healthy controls. Two-sample $t$-tests were used to compare the intergroup differences in CBF and ALFF. Multiple comparisons were corrected using a voxel-wise false discovery rate (FDR) method $(P<0.05)$. Results. All hemodialysis patients and healthy controls showed significant across-voxel correlations between CBF and ALFF. Hemodialysis patients showed a significantly reduced global CBF-ALFF coupling ( $P=0.0011)$ compared to healthy controls at the voxel-level. Of note, decreased CBF/ALFF ratio was exclusively located in the bilateral amygdala involved in emotional regulation and cognitive processing in hemodialysis patients. In hemodialysis patients, the decreased CBF (right olfactory cortex, anterior cingulate gyrus and bilateral insula) and ALFF (bilateral precuneus and superior frontal gyrus) were mainly located in the default mode network (DMN) and salience network-related regions as well as increased CBF in the bilateral thalamus. Conclusions. These novel findings reveal that disrupted neurovascular coupling may be a potential neural mechanism in hemodialysis patients. 


\section{Disturbed neurovascular coupling in hemodialysis patients}

2

3 Mei Jin ${ }^{1}$, Liyan Wang ${ }^{2}$, Hao Wang ${ }^{1}$, Xue Han ${ }^{2}$, Zongli Diao ${ }^{2}$, Wang Guo ${ }^{2}$, Zhenghan Yang ${ }^{1}$,

4 Heyu Ding ${ }^{1}$, Zheng Wang ${ }^{1}$, Peng Zhang ${ }^{1}$, Pengfei Zhao ${ }^{1}$, Han Lv ${ }^{1}$, Wenhu Liu ${ }^{2}$, Zhenchang

5 Wang $^{1 *}$

6

$7{ }^{1}$ Department of Radiology, Beijing Friendship Hospital, Capital Medical University, Beijing,

8 China

92 Department of Nephrology, Faculty of Kidney Diseases, Beijing Friendship Hospital, Capital

10 Medical University, Beijing, China

12 Corresponding Author:

13 Zhenchang Wang ${ }^{1}$

14 No. 95 Yong An Road, Xicheng District, Beijing, 100050, China

Email address: cjr.wzhch@vip.163.com 


\section{Abstract}

Background. Altered cerebral blood flow (CBF) and amplitude of low-frequency fluctuation

(ALFF) have been reported in hemodialysis patients. However, neurovascular coupling impairments, which provide a novel insight into the human brain, have not been reported in hemodialysis patients.

Methods. We combined arterial spin labeling (ASL) and blood oxygen level dependent (BOLD) techniques to investigate neurovascular coupling alterations and its relationships with demographic and clinical data in 46 hemodialysis patients and 47 healthy controls. To explore regional neuronal activity, ALFF was obtained from resting-state functional MRI. To measure cerebral vascular response, $\mathrm{CBF}$ was calculated from ASL. The across-voxel CBF-ALFF correlations for global neurovascular coupling and CBF/ALFF ratio for regional neurovascular coupling were compared between hemodialysis patients and healthy controls. Two-sample $t$-tests were used to compare the intergroup differences in CBF and ALFF. Multiple comparisons were corrected using a voxel-wise false discovery rate $($ FDR $)$ method $(P<0.05)$.

Results. All hemodialysis patients and healthy controls showed significant across-voxel correlations between CBF and ALFF. Hemodialysis patients showed a significantly reduced 
43 global CBF-ALFF coupling $(P=0.0011)$ compared to healthy controls at the voxel-level. Of

44 note, decreased $\mathrm{CBF} / \mathrm{ALFF}$ ratio was exclusively located in the bilateral amygdala involved in

45 emotional regulation and cognitive processing in hemodialysis patients. In hemodialysis patients,

46 the decreased CBF (right olfactory cortex, anterior cingulate gyrus and bilateral insula) and

47 ALFF (bilateral precuneus and superior frontal gyrus) were mainly located in the default mode

48 network (DMN) and salience network-related regions as well as increased CBF in the bilateral

49 thalamus.

50 Conclusions. These novel findings reveal that disrupted neurovascular coupling may be a

51 potential neural mechanism in hemodialysis patients.

\section{Introduction}

55 End-stage renal disease (ESRD) is the final stage of chronic kidney disease, and diagnosed as the estimated glomerular filtration rate $<15 \mathrm{ml} / \mathrm{min} / 1.7 \mathrm{~m}^{2}$ or the permanent functional point $<10 \%$ of kidney capacity. ESRD is often accompanied by multiple-organ dysfunction, especially central nervous system and neurological symptoms (Brouns \& De Deyn 2004; Foley \& Collins 2007). There is high prevalence of cognitive dysfunction among hemodialysis patients compared with healthy controls (Bugnicourt et al. 2013; Kurella Tamura \& Yaffe 2011). Cognitive impairments (global cognition, attention, executive function and memory) may reduce the ability of patients in hemodialysis care, such as decreased medication adherence or lower life quality

63 (Agganis et al. 2010; Cukor et al. 2009). Moreover, cognitive impairment is a common 
64

65

66

67

68

69

complication in ESRD patients with hemodialysis, leading to higher mortality and costly care (Agganis et al. 2010; Chen et al. 2015b). However, the mechanisms of neuro-pathophysiology of cognitive dysfunction in ESRD patients with hemodialysis remain largely unknown. Consequently, understanding neuropathological mechanisms of cognitive impairment in hemodialysis patients is crucial for improvement of diagnosis, treatment and follow-up. Resting-state functional MRI is a useful tool for identifying functional abnormalities of cognitive complications in hemodialysis patients by analyzing blood oxygen level dependent (BOLD) signals. For example, amplitude of low-frequency fluctuation (ALFF) (Luo et al. 2016) and regional homogeneity (Chen et al. 2015a) have been used to reveal spontaneous neuronal activity in ESRD patients. More recently, increasing evidence (Ma et al. 2016; Zhang et al. 2016) of disrupted resting-state brain network has been consistently detected in ESRD patients according to the methods of independent component analysis or nodal centrality (Li et al. 2016; Ling et al. 2014; Ma et al. 2015). The default mode network (DMN) is believed to be a wellestablished network in resting state, and typically includes the key nodes of the anterior cingulate and medial prefrontal cortex, posterior cingulate cortex and precuneus. Taken together, these core regions are involved in cognitive processing of the regulation between cognition and emotion, self-relevance, memory encoding, consolidation, motor activity and mind wandering (Buckner et al. 2008; Greicius et al. 2003; van den Heuvel \& Hulshoff Pol 2010). The salience network, comprised of dorsal anterior cingulate cortex and anterior insula, contributes to cognitive and behavioral control and participates in processing of directing attention (Liang et al. 2015; Shi et al. 2019). Furthermore, evidence from hemodialysis patients has shown altered 
85

86

87

amygdala-related functional connectivity (Chen et al. 2017; Li et al. 2018). The amygdala plays a critical role in interactive processing of affective and cognitive networks based on shared underlying neuro-circuitry (Mechelli et al. 2005; Soriano-Mas et al. 2013; Wu et al. 2017). Simultaneously, brain vascular supply can be assessed by cerebral blood flow (CBF) computed from arterial spin labeling (ASL) (Zheng et al. 2016). Using endogenous contrast, ASL is a noninvasive technique that can quantify $\mathrm{CBF}$ in a given time and explore the altered $\mathrm{CBF}$ in hemodialysis patients. Hemodialysis patients exhibited increased or decreased CBF in widespread regions of the brain, particularly decreased CBF in the prefrontal cortex (Cheng et al. 2019; Papoiu et al. 2014).

The human brain, which accounts for only $2 \%$ of body weight, consumes nearly $20 \%$ oxygen and glucose of the whole body, supporting spontaneous brain activity at resting-state (Phillips et al. 2016). As expected, there was a close relationship between CBF and brain metabolism, including glucose utilization, oxygen consumption, and aerobic glycolysis (Vaishnavi et al. 2010). Altered cerebral perfusion or neuronal activity in cerebral cortex was detected using only unimodal neuroimaging technique in several previous studies (Luo et al. 2016; Peng et al. 2018). However, these results could not fully reflect their close coupling situations. Thus, multimodal neuroimaging techniques were expected to detect cerebral coupling abnormalities in hemodialysis patients in this study. Several studies have investigated the close neurovascular coupling between CBF and neuronal activity in the normal brain (Phillips et al. 2016) and abnormal pathological conditions including Alzheimer's disease (Tarantini et al. 2017), neuromyelitis optica (Guo et al. 2019), schizophrenia (Zhu et al. 2017) and type 2 diabetes 
106

107

108

109

110

111

112

113

114

115

116

117

118

119

120

121

122

123

124

125

126

Peer) reviewing PDF | (2019:12:44117:2:0:NEW 20 Mar 2020)

mellitus (Hu et al. 2019).

\section{Materials and Methods}

\section{Participants}

In our study, BOLD data were used to measure ALFF value, and ASL perfusion data were explored to measure CBF value. Thus, we used ALFF to represent brain neuronal activity and CBF to reflect brain vascular response. Given the spatially different distribution of CBF and ALFF of the brain in hemodialysis patients and healthy controls, we hypothesized that hemodialysis patients could reveal a decreased global CBF-ALFF coupling compared with healthy subjects. Owing to the different affected regions in CBF and ALFF of the brain, we also hypothesized that hemodialysis patients could have specific affected regions of the CBF/ALFF ratio. Furthermore, we hypothesized that brain regions with increased/unchanged CBF and decreased ALFF could show an increased CBF/ALFF ratio, while brain regions with decreased/unchanged CBF and increased ALFF could show a decreased CBF/ALFF ratio in hemodialysis patients. To address these hypotheses, we collected ASL perfusion and restingstate BOLD data from 46 hemodialysis patients and 47 healthy controls. Global CBF-ALFF coupling analysis in gray matter and regional CBF/ALFF ratio analysis of each voxel were performed to compare the differences between hemodialysis patients and healthy controls. The results of this study are expected to provide an objective imaging biomarker and facilitate understanding of the neurovascular mechanism in hemodialysis patients from a new perspective.

The study protocol was approved by the Medical Research Ethics Committee of the Beijing Friendship Hospital (Approval Number: 2018-P2-158-02) and signed informed consent forms 
127 were obtained from all participants. A total of 111 right-handed participants (age $\geq 18$ years)

128 were enrolled in this study, including 58 patients with hemodialysis for at least six months (Liu

129 et al. 2016b; Yen et al. 2009) from the Department of Nephrology of Beijing Friendship Hospital

130 and 53 healthy controls from the local community. The exclusion criteria were subjects with

131 neuropsychiatric history, benign or malignant brain tumor history, cerebrovascular disease, a

132 history of head trauma, other systemic diseases, renal transplant history, MRI contraindications,

133 head motion, and poor imaging quality.

134 Demographic data including age and gender and hemodialysis times of each patient were

retrieved and reviewed from the electronic medical records system. The measurements of

brachial artery blood pressure (BP) of hemodialysis patients were performed at rest just before

MRI scans and the average BP value of three consecutive measurements over ten minutes was

recorded.

\section{Laboratory Examinations}

Blood specimens of hemodialysis patients were drawn from the arterial end of the vascular

access and were collected on the inter-dialytic (stable hemodialysis sessions) day before MRI

scans to minimize the effect of potential bias. Serum levels of hemoglobin $(\mathrm{g} / \mathrm{l})$, creatinine

$(\mu \mathrm{mol} / \mathrm{l})$, serum calcium and phosphorus $(\mathrm{mmol} / \mathrm{l})$, albumin $(\mathrm{g} / \mathrm{l})$, blood urea nitrogen $(\mathrm{mmol} / \mathrm{l})$

and serum ferritin $(\mathrm{ng} / \mathrm{ml})$ were measured and analyzed at the clinical laboratory of Beijing

Friendship Hospital, with standard laboratory procedures using an automatic analyzer. The 
148 149 150

40.00-55.00 g/1, 2.60-7.50 mmol/1 and 24.00-336.00 ng/ml in Beijing Friendship Hospital. No laboratory examination was performed in healthy controls.

\section{Data Acquisition}

All imaging studies were performed at the Department of Radiology of Beijing Friendship Hospital. All MRI data were acquired using a 3.0-Tesla MR scanner (Discovery MR750, General Electric, Milwaukee, WI, USA) equipped with an eight-channel, phased-array head coil.

Earplugs were used for noise reduction, and tight but comfortable sponge pads were used to minimize head motion. All subjects were asked to remain still, thoughtless and relaxed, keep their eyes closed, and stay awake during the scans.

Resting-state perfusion data were acquired by using a pseudo-continuous ASL sequence with background suppression. The parameters were as follows: repetition $(\mathrm{TR})=4852 \mathrm{~ms}$; echo time $(\mathrm{TE})=10.7 \mathrm{~ms}$; field of view $(\mathrm{FOV})=24 \mathrm{~cm} \times 24 \mathrm{~cm}$; matrix $=128 \times 128$; flip angle $(\mathrm{FA})=$ $111^{\circ}$; slice thickness $=4.0 \mathrm{~mm}$; no gap; 36 slices; post label delay $=2025 \mathrm{~ms}$; number of excitation $=3$. Resting-state BOLD data were acquired with the following parameters: $\mathrm{TR}=$ $2000 \mathrm{~ms} ; \mathrm{TE}=35 \mathrm{~ms} ; \mathrm{FOV}=24 \mathrm{~cm} \times 24 \mathrm{~cm} ;$ matrix $=64 \times 64 ; \mathrm{FA}=90^{\circ} ;$ slice thickness $=5.0$ mm; gap $=1 \mathrm{~mm} ; 28$ slices; 200 time-points. High-resolution three-dimensional T1-weighted images had the following parameters: $\mathrm{TR}=8.492 \mathrm{~ms} ; \mathrm{TE}=3.276 \mathrm{~ms}$; inversion time $(\mathrm{TI})=450$ $\mathrm{ms} ; \mathrm{FOV}=24 \mathrm{~cm} \times 24 \mathrm{~cm} ;$ matrix $=256 \times 256 ; \mathrm{FA}=15^{\circ} ;$ slice thickness $=1.0 \mathrm{~mm}$; no gap; 196 slices.

\section{Cerebral Blood Flow Preprocessing}

Using a single compartment model (Buxton et al. 1998), ASL difference images were computed 
169

170

171

172

173

174

175

176

177

178

after the deduction of the label images from the control images. The CBF maps were calculated from the combination with the ASL difference images and the proton density-weighted reference images (Xu et al. 2010). The detailed steps of CBF preprocessing were as follows (Liu et al. 2016a). 1) Statistical Parametric Mapping (SPM8, https://www.fil.ion.ucl.ac.uk/spm/) software was used to normalize the CBF images to the standard Montreal Neurological Institute (MNI) space. 2) Individual ASL difference images of healthy controls were nonlinearly co-registered to the standard perfusion template in MNI space, and then averaged to generate a study-specific ASL template. 3) All individual ASL difference images of every subject were nonlinearly coregistered to this study-specific ASL template. 4) All CBF images were written into the standard space with the normalization parameters (derived from the previous step) and resliced into a $2 \times$ $2 \times 2 \mathrm{~mm}^{3}$ cubic voxel. 5) The co-registered CBF maps were removed of non-brain tissue and divided by the global mean $\mathrm{CBF}$ value of the gray matter. 6) The standardized images were spatially smoothed with a Gaussian kernel of $6 \times 6 \times 6 \mathrm{~mm}^{3}$ full-width at half maximum (FWHM).

\section{Functional MRI Data Preprocessing}

The functional MRI data preprocessing was performed in Data Processing Assistant for RestingState Functional (DPARSF, http://rfmri.org/DPARSF) MRI software package. In total, 190 volumes (200 volumes were acquired, and the first 10 volumes were discarded for signal stabilization) were used for functional imaging data preprocessing. The following steps comprised slice timing, realignment, nuisance regressions, spatial normalization, and smoothening (a $6 \times 6 \times 6 \mathrm{~mm}^{3}$ FWHM Gaussian kernel): 1) Slice time correction for the timing 
190

191

192

193

194

195

196

197

198

199

200

201

202

203

204

205

206

207

208

209

210

differences between slices and realignment for head-motion correction were performed. Head-

motion thresholds were less than $3.0 \mathrm{~mm}$ of translation or $3.0^{\circ}$ of rotation in any direction.

Frame-wise displacement (FD) was also calculated to exclude the subjects with mean FD larger

than $0.3 \mathrm{~mm}$. No significant difference $(P=0.8138)$ was found in mean FD between the

hemodialysis patients $(0.0521 \pm 0.0062)$ and healthy controls $(0.0504 \pm 0.0039)$. Then, mean FD

was included as a covariate for the next statistical step. 2) The nuisance regressions (motion

parameters based on the Friston-24 model, the average signals of the white matter and

ventricular) were extracted as covariates. 3) In the normalization step, individual structural

images were co-registered to the mean functional image; the transformed structural images were

then segmented and spatially normalized to the MNI space using the Diffeomorphic Anatomical

Registration Through the Exponentiated Lie algebra (DARTEL) technique (Ashburner 2007).

For the ALFF analysis, the time course of each voxel was converted to the frequency domain

using a Fast Fourier Transform. The averaged square root of the power spectrum (ALFF) value

was then calculated across $0.01-0.08 \mathrm{~Hz}$ for all voxels in each participant. Finally, the ALFF

value of each voxel was divided by the global brain mean ALFF value for standardization

procedure (Liu et al. 2013).

\section{Global CBF-ALFF Coupling Analysis}

For each subject, Fisher's z transformation was applied to both CBF and ALFF images for improving the normality of the data distribution and comparing across participants (Liu et al.

2017). The specific statistical processing was adopted from a previous study (Liang et al. 2013).

For the global neurovascular coupling between CBF and ALFF, correlation analyses across 
211 voxels were performed for each subject. Because the neighboring voxels would be highly

212 dependent due to spatial preprocessing such as registration and spatial smoothing, the effective

213 degree of freedom $\left(d f_{e f f}\right)$ in across-voxel correlation analyses would be much smaller than the

214 number of voxels in the gray matter mask. Consequently, the $d f_{\text {eff }}$ in across-voxel correlation

215 analyses was estimated using the following equation:

216

$$
d f_{e f f}=\frac{N}{(\text { FWHMx } \times \text { FWHMy } \times \mathrm{FWHMz}) / v}-2
$$

217 where $v$ is the volume of a voxel $\left(2 \times 2 \times 2 \mathrm{~mm}^{3}\right)$ and $N$ is the number of voxels $(N=163735)$

218 used in the analyses. FWHM $\times$ $\times$ FWHMy $\times$ FWHMz as the average smoothness of the CBF and

219 ALFF images $\left(9.7 \times 10.7 \times 10.2 \mathrm{~mm}^{3}\right)$ was estimated using the software RESTplus

220 (http://www.restfmri.net/). Therefore, the $d f_{\text {eff }}$ in across-voxel correlations was 1235 in the

221 present study. Consequently, a CBF-ALFF correlation coefficient value for each participant

222 reflected the spatially consistent distribution between CBF and ALFF at gray matter level. Then,

223 a two-sample $t$-test was used to compare the intergroup difference in CBF-ALFF correlation

224 coefficients.

225 Regional CBF/ALFF Ratio Analysis

226 For the regional neurovascular coupling (blood supply per unit of neuronal activity), the

227 CBF/ALFF ratio of each voxel was calculated for each subject. To identify significant difference

228 in brain regions between the hemodialysis and healthy groups, a two-sample $t$-test was used to

229 compare the differences in CBF/ALFF ratio, with age and gender as covariates. Multiple

230 comparisons were performed by a voxel-wise false discovery rate (FDR) correction, with a

231 corrected threshold of $P<0.05$. 
232 Voxel-wise Comparisons in CBF and ALFF

233 To better understand the differences in CBF/ALFF ratio, voxel-wise comparisons were

234 performed to detect the CBF and ALFF differences between the hemodialysis and healthy groups,

235 by controlling age and gender as covariates. Multiple comparisons were also corrected using a

236 voxel-wise FDR method $(P<0.05)$.

237 Clinical Correlation Analysis

238 Significant regions were extracted from regional CBF/ALFF ratio analysis and voxel-wise

239 comparisons in CBF and ALFF between the two groups. Pearson's correlation analyses were

240 then performed to evaluate correlations between these regions and clinical variables (including

241 hemodialysis times, blood pressure, hemoglobin, creatinine, calcium, phosphorus, albumin,

242 blood urea nitrogen and serum ferritin) in the hemodialysis group. A $P$ value $<0.05$ was

243 considered for significant differences.

244 Statistical Analysis

245 Two-sample $t$-test was used to detect difference in age between the hemodialysis group and the

246 control group. Chi-square test was used to demonstrate difference in gender between the two

247 groups. All statistical analyses were performed using SPSS (an IBM Company, Chicago, USA).

248 Statistical significance was set as $P<0.05$.

\section{Results}

250 Of the 111 participants (58 hemodialysis patients and 53 healthy controls), 18 participants were excluded due to the following reasons: one hemodialysis patient (left middle cerebral artery occlusion) and one healthy control (arteriovenous malformation) with cerebrovascular disease; 
253 one healthy control with benign brain tumor (cavernous hemangioma); six hemodialysis patients

254 and one healthy control with head motion greater than $3.0 \mathrm{~mm}$ or $3.0^{\circ}$ in any direction and three

255 hemodialysis patients and three healthy controls with mean FD larger than $0.3 \mathrm{~mm}$; two

256 hemodialysis patients with claustrophobia. Finally, 46 hemodialysis patients and 47 healthy

257 controls were included for analysis in this study.

258 Demographic Data and Clinical Variables

259 The characteristic demographic data of hemodialysis patients and healthy controls were

260 presented in Table 1. No significant differences were observed in age and gender between the

261 two groups. The results of hemodialysis times, blood pressure and blood tests of the

262 hemodialysis patients were also shown in Table 1.

263 Global CBF-ALFF Coupling Changes in Hemodialysis Patients

264 Hemodialysis patients showed a significantly reduced global CBF-ALFF coupling $(P=0.0011)$

265 compared to healthy controls (Figure 1). Significant difference was observed between two

266 groups on CBF-ALFF correlation coefficients across voxels. In addition, all hemodialysis

267 patients and all healthy controls showed significant across-voxel correlations between CBF and

268 ALFF.

269 Spatial Distribution of the CBF/ALFF Ratio

270 The spatial distribution maps of CBF/ALFF ratio in healthy controls (Figure 2A) and

271 hemodialysis patients (Figure 2B) were shown in Figure 2. Although somewhat differences

272 were found in brain regions and magnitudes, the two groups exhibited relatively consistent

273 spatial distribution in these measures.

Peer] reviewing PDF | (2019:12:44117:2:0:NEW 20 Mar 2020) 
274

275

276

277

278

279

280

281

282

283

284

285

286

287

288

289

290

291

292

293

294

\section{Regional CBF/ALFF Ratio Changes in Hemodialysis Patients}

Compared to healthy controls, hemodialysis patients showed decreased CBF/ALFF ratio in left

(Figure 3A-C) and right (Figure 3D-F) amygdala $(P<0.05$, voxel-wise FDR correction). No significantly increased different regions were observed in regional CBF/ALFF ratio between two groups.

\section{CBF and ALFF Changes in Hemodialysis Patients}

Compared with healthy controls, hemodialysis patients showed increased CBF in the thalamus

bilaterally (Figure 4A-F) as well as decreased CBF in the right olfactory cortex and bilateral insula and anterior cingulated gyrus (Figure 4G-L) $(P<0.05$, voxel-wise FDR correction)

(Figure 4; Table 2). Compared with healthy controls, hemodialysis patients exhibited increased

ALFF in the amygdala bilaterally (Figure 5A-F) as well as decreased ALFF in superior frontal gyrus and precuneus bilaterally (Figure 5G-L) $(P<0.05$, voxel-wise FDR correction) (Figure 5;

\section{Table 3).}

\section{Correlation Analysis of Clinical Variables}

No significant difference was found in the correlations between each significant region and clinical variables (blood tests and hemodialysis times) in the hemodialysis and healthy groups $(P>$ $0.05)$.

\section{Discussion}

To the best our knowledge, this is the first study to explore CBF-ALFF coupling alterations in hemodialysis patients by combining ASL and BOLD techniques. Compared with healthy controls, hemodialysis patients showed reduced across-voxel CBF-ALFF correlations of global 
295

296

297

298

299

300

301

302

303

304

305

306

307

308

309

310

311

312

313

314

315

neurovascular coupling. Moreover, hemodialysis patients revealed decreased CBF/ALFF ratio in the bilateral amygdala, which was involved in the emotion- and cognition- related regions.

Furthermore, hemodialysis patients showed decreased CBF and ALFF bilaterally in DMNrelated regions as well as increased $\mathrm{CBF}$ in the bilateral thalamus. These findings may provide objective neuroimaging evidence and improve the understanding of neural mechanisms from the perspective of neurovascular coupling in hemodialysis patients.

Consistent with our hypothesis, we found a significant across-voxel correlation between CBF and ALFF in healthy subjects, which was also reported in previous studies (Guo et al. 2019; Liang et al. 2013; Zhu et al. 2017), indicating the physiological neurovascular coupling in healthy brain. Additionally, across-voxel correlation between CBF and ALFF was also observed in hemodialysis patients, which was lower than that in healthy controls at the global level, presumably indicating global neurovascular decoupling in hemodialysis patients. Two possible explanations for global neurovascular decoupling in hemodialysis patients were as follows: 1) Chronic kidney disease has an impact on cerebrovascular hemodynamic alterations by changing CBF (Liu et al. 2018; Sedaghat et al. 2016). The changed CBF can lead to altered cerebral oxygen saturation, which is affected by hemodialysis duration, serum albumin concentration, and blood pH (Ito et al. 2015). Moreover, vessel impairment due to CBF alterations can lead to neurovascular decoupling. 2) Cerebral autoregulation, which is a complex control mechanism, maintains relatively constant $\mathrm{CBF}$ via changing cerebral vascular resistance in response to alterations of cerebral perfusion pressure or metabolic needs (Ghoshal \& Freedman 2019). The neurovascular coupling relies on the integrity of neurovascular unit, whose components are 
316

317

318

neurons, astrocytes, and vasculature (Muoio et al. 2014). In this unit, astrocytes may play an important role in information exchange between neurons and vessels (Howarth 2014; JL \& CM 2013), breaking the balance between neuronal activity and blood supply (Filosa et al. 2016). However, the impairment of neurovascular coupling in hemodialysis patient needs to be further explored.

Hemodialysis patients showed decreased CBF/ALFF ratio in the bilateral amygdala, which were the key nodes in emotional regulation and cognitive processing (Chen et al. 2017). In the present study, the bilateral amygdala showed normal CBF and increased ALFF, suggesting that the $\mathrm{CBF} / \mathrm{ALFF}$ ratio reduction was mainly driven by the increased ALFF. This abnormality may at least partly account for the emotional and cognitive dysfunction in hemodialysis patients. The $\mathrm{CBF} / \mathrm{ALFF}$ ratio maintains the balance between two aspects in the healthy brain (Guo et al. 2019). One aspect is vascular response and the other aspect is neuronal activity. The disruption in the $\mathrm{CBF} / \mathrm{ALFF}$ ratio balance may lead to regional $\mathrm{CBF} / \mathrm{ALFF}$ ratio enhancement or reduction. The increased $\mathrm{CBF} / \mathrm{ALFF}$ ratio indicates redundant CBF per unit of neuronal activity or decreased ALFF with normal blood supply, whereas the decreased CBF/ALFF ratio indicates inadequate CBF per unit of neuronal activity or increased ALFF with normal blood supply. The deviation from the CBF/ALFF ratio balance in hemodialysis patients in this study is consistent with the latter situation. Moreover, the analysis of CBF/ALFF ratio can identify abnormal regions without significant intergroup differences in CBF or ALFF. Impairments of the amygdala in hemodialysis have been previously reported. One study (Li et al. 2018) combined structural covariance and functional connectivity analyses, and revealed amygdala-related pattern 
337 alterations of structural covariance and functional connectivity. The results demonstrated the

338 reciprocal emotion-cognition interaction through amygdala-related emotional control network in

339 hemodialysis patients. The authors also found that the hemoglobin level could directly lead to

340 abnormal functional connectivity between amygdala and anterior cingulate cortex. Another study

341 (Chen et al. 2017) showed a correlation between aberrant amygdala-based emotional regulatory

342 circuits and depression in hemodialysis patients. Impaired neurocognitive performance was

343 ascribed to the amygdala-related specific region in the hemodialysis patients. Nevertheless, the

$344 \mathrm{CBF} / \mathrm{ALFF}$ ratio is a new approach to measure regional alterations of neurovascular coupling in

345 hemodialysis patients, and should be further investigated in the future.

346 Specifically, this study found significant intergroup differences in CBF and ALFF by voxel-

347 wise analyses in several brain regions between hemodialysis patients and healthy controls. For

348 example, hemodialysis patients exhibited decreased CBF in the right olfactory cortex and

349 anterior cingulate gyrus, and decreased ALFF in the bilateral precuneus and superior frontal

350 gyrus, most of which were components of the DMN. Several DMN-related regions showed

351 decreased CBF in the inferior and middle frontal lobes, inferior temporal and parietal lobes

352 (Cheng et al. 2019; Papoiu et al. 2014), decreased regional homogeneity in the bilateral frontal,

353 parietal and temporal lobes (Chen et al. 2015a), decreased gray matter volume in the bilateral

354 precuneus and right frontal lobe (Zhang et al. 2013), reported by previous studies. Moreover,

355 DMN-related decreases of functional connectivity have also been identified in hemodialysis

356 patients, such as between medial prefrontal cortex and posterior cingulate cortex and precuneus

357 (Ling et al. 2014). The DMN, which is a well-established network, has high metabolic activity at

Peer) reviewing PDF | (2019:12:44117:2:0:NEW 20 Mar 2020) 
358

359

360

361

362

363

364

365

366

367

368

369

370

371

372

373

374

375

376

377

378

rest and is restrained during tasks (Buckner et al. 2008). The DMN contributes to memory encoding, environmental monitoring, self-relevance and social functions, and regulates the interaction between cognitive functions and emotional processes (Greicius et al. 2003; Raichle \& Snyder 2007). Meanwhile, decreased CBF in the bilateral insular and anterior cingulate gyrus was identified in the hemodialysis group as compared to the healthy controls. Of note, the salience network typically comprises the bilateral insular and anterior cingulate gyrus. The insular, which is the key hub of the salience network, contributes to attentional, cognitive and behavioral functions (Menon \& Uddin 2010) and pain perception (Brooks \& Tracey 2007). The anterior cingulate gyrus, which is considered as a part of the cerebral limbic system, participates in emotional regulation (Bush et al. 2000), visual and auditory attention, language processing, memory, and motor activity (Garrity et al. 2007). Furthermore, increased CBF was found in the bilateral thalamus of hemodialysis patients relative to healthy controls. The thalamus is regarded as a crucial relay station of multiple functional circuits regulating cognitive processing, including memory, emotion, attention and information processing (Fama \& Sullivan 2015). Taken together, DMN, salience network and thalamus were impaired in hemodialysis patients, which might explain the aberrant neurocognitive performance of these patients.

No significant difference was found in the correlations between each significant region and clinical variables (blood tests and hemodialysis times) in the hemodialysis and healthy groups. However, only hemoglobin levels were slightly below the normal range, while creatinine and blood urea nitrogen levels were substantially higher than the normal range due to the final stage of chronic kidney disease. Hemodialysis patients always suffer from anemia, malnutrition and 
379

380

381

382

383

384

385

protein-energy wasting conditions resulting from different pathogenic causes. Previous studies have shown that hemodialysis patients were usually vulnerable to under-nutrition caused by insufficient nutrient supply to the brain, resulting in brain abnormalities, such as cerebral hypoxia, decreased blood viscosity, brain hypo-perfusion and/or hypo-metabolism (Christina et al. 2012; Yasuo et al. 2002). Therefore, long-term hemodialysis can significantly affect the cerebral circulation, function and metabolism, leading to poor mental health of hemodialysis patients due to low hemoglobin levels (Christina et al. 2012). Other laboratory biochemical indicators exhibited a relatively normal range, and it was speculated that hemodialysis patients on regular use of vitamin $\mathrm{D}$, calcitriol and/or phosphorus-chelating agents might have contributed to the steady state noted in the present study.

This study had several limitations. First, neuropsychological tests were not conducted to confirm different symptoms, so the relationship between the alterations of CBF/ALFF coupling and neurological complications could not be clarified, and the possibility of heterogeneity in hemodialysis patients could not be ruled out, which may affect the results. Second, CBF and ALFF indirectly reflect vascular response and neuronal activity, respectively. Thus, the analyses of the across-voxel CBF-ALFF correlations and CBF/ALFF ratio were indirect measurements of neurovascular coupling in hemodialysis patients. The technical limits may restrict the calculation of exact CBF and ALFF that result in alterations of neurovascular coupling in hemodialysis patients. Third, validation analyses were not performed in the present study. A larger sample size is required in further studies to validate the findings of the current study.

\section{Conclusions}


400 This study revealed neurovascular coupling impairments in hemodialysis patients via a

401 combination of ASL and BOLD techniques. Specifically, decreased CBF/ALFF ratio in the

402 bilateral amygdala was involved in emotional regulation and cognitive processing in

403 hemodialysis patients. In addition, the DMN, salience network and thalamus were also impaired

404 in hemodialysis patients, which might explain the aberrant neurocognitive performance of these

405 patients. Consequently, these findings presented novel evidence that disrupted neurovascular

406 coupling may be a potential neural mechanism in hemodialysis patients.

407

408

References

409

410

411

412

413

414

415

416

417

418

419

420

Agganis BT, Weiner DE, Giang LM, Scott T, Tighiouart H, Griffith JL, and Sarnak MJ. 2010. Depression and cognitive function in maintenance hemodialysis patients. American Journal of Kidney Diseases 56:704-712. 10.1053/j.ajkd.2010.04.018.

Ashburner J. 2007. A fast diffeomorphic image registration algorithm. Neuroimage 38:95-113. 10.1016/j.neuroimage.2007.07.007.

Brooks JC, and Tracey I. 2007. The insula: a multidimensional integration site for pain. Pain 128:1-2. 10.1016/j.pain.2006.12.025.

Brouns R, and De Deyn PP. 2004. Neurological complications in renal failure: a review. Clinical Neurology and Neurosurgery 107:1-16. 10.1016/j.clineuro.2004.07.012.

Buckner RL, Andrews-Hanna JR, and Schacter DL. 2008. The brain's default network: anatomy, function, and relevance to disease. Annals of New York Academy of Sciences 1124:1-38. 10.1196/annals.1440.011. 
421

422

423

424

425

426

427

428

429

430

431

432

433

434

435

436

437

438

439

440

441

Bugnicourt JM, Godefroy O, Chillon JM, Choukroun G, and Massy ZA. 2013. Cognitive disorders and dementia in CKD: the neglected kidney-brain axis. Journal of the American Society of Nephrology 24:353-363. 10.1681/ASN.2012050536.

Bush G, Luu P, and Posner MI. 2000. Cognitive and emotional influences in anterior cingulate cortex. Trends in Cognitive Sciences 4:215-222. 10.1016/s1364-6613(00)01483-2.

Buxton RB, Frank LR, Wong EC, Siewert B, Warach S, and Edelman RR. 1998. A general kinetic model for quantitative perfusion imaging with arterial spin labeling. Magnetic Resonance in Medicine 40:383-396. 10.1002/mrm.1910400308.

Chen HJ, Qi R, Kong X, Wen J, Liang X, Zhang Z, Li X, Lu GM, and Zhang LJ. 2015a. The impact of hemodialysis on cognitive dysfunction in patients with end-stage renal disease: a resting-state functional MRI study. Metabolic Brain Disease 30:1247-1256. 10.1007/s11011-015-9702-0.

Chen HJ, Wang YF, Qi R, Schoepf UJ, Varga-Szemes A, Ball BD, Zhang Z, Kong X, Wen J, Li X, Lu GM, and Zhang LJ. 2017. Altered Amygdala Resting-State Functional Connectivity in Maintenance Hemodialysis End-Stage Renal Disease Patients with Depressive Mood. Molecular Neurobiology 54:2223-2233. 10.1007/s12035-016-9811-8.

Chen HJ, Zhang LJ, and Lu GM. 2015b. Multimodality MRI Findings in Patients with End-Stage Renal Disease. Biomed Research International 2015:697402. 10.1155/2015/697402.

Cheng BC, Chen PC, Chen PC, Lu CH, Huang YC, Chou KH, Li SH, Lin AN, and Lin WC. 2019. Decreased cerebral blood flow and improved cognitive function in patients with end-stage renal disease after peritoneal dialysis: An arterial spin-labelling study. 
443 Christina B, Georgios A, Ioannis G, Christos P, and Polichronis A. 2012. Relationship between

444

445

446

447

448

449

450

451

452

453

454

455

456

457

458

459

460

461

462

depression, clinical and biochemical parameters in patients undergoing haemodialysis.

Journal of Renal Care 38:93-97. 10.1111/j.1755-6686.2012.00259.x.

Cukor D, Rosenthal DS, Jindal RM, Brown CD, and Kimmel PL. 2009. Depression is an important contributor to low medication adherence in hemodialyzed patients and transplant recipients. Kidney International 75:1223-1229. 10.1038/ki.2009.51.

Fama R, and Sullivan EV. 2015. Thalamic structures and associated cognitive functions:

Relations with age and aging. Neuroscience and Biobehavioral Reviews 54:29-37. 10.1016/j.neubiorev.2015.03.008.

Filosa JA, Morrison HW, Iddings JA, Du W, and Kim KJ. 2016. Beyond neurovascular coupling, role of astrocytes in the regulation of vascular tone. Neuroscience 323:96-109.

10.1016/j.neuroscience.2015.03.064.

Foley RN, and Collins AJ. 2007. End-stage renal disease in the United States: an update from the United States Renal Data System. Journal of the American Society of Nephrology 18:2644-2648. 10.1681/ASN.2007020220.

Garrity AG, Pearlson GD, McKiernan K, Lloyd D, Kiehl KA, and Calhoun VD. 2007. Aberrant "default mode" functional connectivity in schizophrenia. American Journal of Psychiatry 164:450-457. 10.1176/ajp.2007.164.3.450.

Ghoshal S, and Freedman BI. 2019. Mechanisms of Stroke in Patients with Chronic Kidney Disease. American Journal of Nephrology 50:229-239. 10.1159/000502446.

Peer) reviewing PDF | (2019:12:44117:2:0:NEW 20 Mar 2020) 
463 Greicius MD, Krasnow B, Reiss AL, and Menon V. 2003. Functional connectivity in the resting 464 brain: a network analysis of the default mode hypothesis. Proceedings of the National Academy of Sciences of the United States of Amecia 100:253-258. 10.1073/pnas.0135058100.

467

468

469

470

471

472

473

474

475

476

477

478

479

480

481

482

483 Frontiers in Neuroscience 8:103. 10.3389/fnins.2014.00103.

Hu B, Yan LF, Sun Q, Yu Y, Zhang J, Dai YJ, Yang Y, Hu YC, Nan HY, Zhang X, Heng CN, Hou JF, Liu QQ, Shao CH, Li F, Zhou KX, Guo H, Cui GB, and Wang W. 2019. Disturbed neurovascular coupling in type 2 diabetes mellitus patients: Evidence from a comprehensive fMRI analysis. Neuroimage Clinical 22:101802. 10.1016/j.nicl.2019.101802.

Ito K, Ookawara S, Ueda Y, Goto S, Miyazawa H, Yamada H, Kitano T, Shindo M, Kaku Y, Hirai K, Yoshida M, Hoshino T, Nabata A, Mori H, Yoshida I, Kakei M, and Tabei K. 2015. Factors affecting cerebral oxygenation in hemodialysis patients: cerebral oxygenation associates with $\mathrm{pH}$, hemodialysis duration, serum albumin concentration, and diabetes mellitus. PloS one 10:e0117474. 10.1371/journal.pone.0117474.

JL S, and CM A. 2013. Multifunctional role of astrocytes as gatekeepers of neuronal energy supply. Frontiers in Cell Neuroscience 7:38. 10.3389/fncel.2013.00038. 
484

485

486

487

488

489

490

491

492

493

494

495

496

497

498

499

500

501

502

503

504

Kurella Tamura M, and Yaffe K. 2011. Dementia and cognitive impairment in ESRD: diagnostic and therapeutic strategies. Kidney International 79:14-22. 10.1038/ki.2010.336.

Li A, Mu J, Huang M, Zhang Z, Liu J, and Zhang M. 2018. Altered amygdala-related structural covariance and resting-state functional connectivity in end-stage renal disease patients. Metabolic Brain Disease 33:1471-1481. 10.1007/s11011-018-0254-y.

Li S, Ma X, Huang R, Li M, Tian J, Wen H, Lin C, Wang T, Zhan W, Fang J, and Jiang G. 2016. Abnormal degree centrality in neurologically asymptomatic patients with end-stage renal disease: A resting-state fMRI study. Clinical Neurophysiology 127:602-609. 10.1016/j.clinph.2015.06.022.

Liang X, He Y, Salmeron BJ, Gu H, Stein EA, and Yang Y. 2015. Interactions between the salience and default-mode networks are disrupted in cocaine addiction. Journal of Neuroscience 35:8081-8090. 10.1523/JNEUROSCI.3188-14.2015.

Liang X, Zou Q, He Y, and Yang Y. 2013. Coupling of functional connectivity and regional cerebral blood flow reveals a physiological basis for network hubs of the human brain. Proceedings of the National Academy of Sciences of the United States of Amecia 110:1929-1934. 10.1073/pnas.1214900110.

Ling N, Jiqiu W, Long Jiang Z, Tong Z, Rongfeng Q, Qiang X, Xue L, Jianhui Z, Gang Z, and Guang Ming L. 2014. Aberrant default-mode functional connectivity in patients with end-stage renal disease: a resting-state functional MR imaging study. Radiology 271:543552. 10.1148/radiol.13130816.

Liu F, Guo W, Liu L, Long Z, Ma C, Xue Z, Wang Y, Li J, Hu M, Zhang J, Du H, Zeng L, Liu Z, 
505

506

507

508

509

510

511

512

513

514

515

516

517

518

519

520

521

522

523

524

525

Wooderson SC, Tan C, Zhao J, and Chen H. 2013. Abnormal amplitude low-frequency

oscillations in medication-naive, first-episode patients with major depressive disorder: a resting-state fMRI study. Journal of Affective Disorders 146:401-406.

10.1016/j.jad.2012.10.001.

Liu F, Wang Y, Li M, Wang W, Li R, Zhang Z, Lu G, and Chen H. 2017. Dynamic functional network connectivity in idiopathic generalized epilepsy with generalized tonic-clonic seizure. Human Brain Mapping 38:957-973. 10.1002/hbm.23430.

Liu F, Zhuo C, and Yu C. 2016a. Altered Cerebral Blood Flow Covariance Network in Schizophrenia. Frontiers in Neuroscience 10:308. 10.3389/fnins.2016.00308.

Liu HS, Hartung EA, Jawad AF, Ware JB, Laney N, Port AM, Gur RC, Hooper SR, Radcliffe J, Furth SL, and Detre JA. 2018. Regional Cerebral Blood Flow in Children and Young Adults with Chronic Kidney Disease. Radiology 288:849-858.

10.1148/radiol.2018171339.

Liu SL, Qi L, Han WQ, Zhu BS, Zhou X, Jiang SS, Chen MF, Li Y, He W, Liu LF, Hu XH, Xie Y, Zeng FH, and Zu XB. 2016b. Shorter hemodialysis duration is a risk factor for the recurrence of urothelial carcinoma of the bladder in patients on maintenance hemodialysis. Clinical and Translational Oncology 18:304-309. 10.1007/s12094-0151368-x.

Luo S, Qi RF, Wen JQ, Zhong JH, Kong X, Liang X, Xu Q, Zheng G, Zhang Z, Zhang LJ, and Lu GM. 2016. Abnormal Intrinsic Brain Activity Patterns in Patients with End-Stage Renal Disease Undergoing Peritoneal Dialysis: A Resting-State Functional MR Imaging 
Study. Radiology 278:181-189. 10.1148/radiol.2015141913.

527

528

529

530

531

532

533

534

535

536

537

538

539

540

541

542

543

544

545

546

Ma X, Jiang G, Li S, Wang J, Zhan W, Zeng S, Tian J, and Xu Y. 2015. Aberrant functional connectome in neurologically asymptomatic patients with end-stage renal disease. PloS one 10:e0121085. 10.1371/journal.pone.0121085.

Ma X, Tian J, Wu Z, Zong X, Dong J, Zhan W, Xu Y, Li Z, and Jiang G. 2016. Spatial Disassociation of Disrupted Functional Connectivity for the Default Mode Network in Patients with End-Stage Renal Disease. PloS one 11:e0161392. 10.1371/journal.pone.0161392.

Mechelli A, Friston KJ, Frackowiak RS, and Price CJ. 2005. Structural covariance in the human cortex. Journal of Neuroscience 25:8303-8310. 10.1523/JNEUROSCI.0357-05.2005.

Menon V, and Uddin LQ. 2010. Saliency, switching, attention and control: a network model of insula function. Brain Structure and Function 214:655-667. 10.1007/s00429-010-0262-0.

Muoio V, Persson PB, and Sendeski MM. 2014. The neurovascular unit - concept review. Acta Physiologica 210:790-798. 10.1111/apha.12250.

Papoiu AD, Emerson NM, Patel TS, Kraft RA, Valdes-Rodriguez R, Nattkemper LA, Coghill RC, and Yosipovitch G. 2014. Voxel-based morphometry and arterial spin labeling fMRI reveal neuropathic and neuroplastic features of brain processing of itch in end-stage renal disease. Journal of Neurophysiology 112:1729-1738. 10.1152/jn.00827.2013.

Peng L, Ding D, Ma XY, Zhang HW, Liu JX, and Ming Z. 2018. Altered intrinsic brain activity and memory performance improvement in patients with end-stage renal disease during a single dialysis session. Brain Imaging and Behavior 12:1640-1649. 10.1007/s11682-018- 
9828-x.

548 Phillips AA, Chan FH, Zheng MM, Krassioukov AV, and Ainslie PN. 2016. Neurovascular coupling in humans: Physiology, methodological advances and clinical implications. Journal of Cerebral Blood Flow Metabolism 36:647-664. 10.1177/0271678X15617954.

551

552

553

554

555

556

557

558

559

560

561

562

563

564

565

566

567 evolving idea. Neuroimage 37:1083-1090. 10.1016/j.neuroimage.2007.02.041.

Sedaghat S, Vernooij MW, Loehrer E, Mattace-Raso FU, Hofman A, van der Lugt A, Franco OH, Dehghan A, and Ikram MA. 2016. Kidney Function and Cerebral Blood Flow: The Rotterdam Study. Journal of the American Society of Nephrology 27:715-721. 10.1681/ASN.2014111118.

Shi Y, Tong C, Zhang M, and Gao X. 2019. Altered functional connectivity density in the brains of hemodialysis end-stage renal disease patients: An in vivo resting-state functional MRI study. PloS one 14:e0227123. 10.1371/journal.pone.0227123.

Soriano-Mas C, Harrison BJ, Pujol J, Lopez-Sola M, Hernandez-Ribas R, Alonso P, ContrerasRodriguez O, Gimenez M, Blanco-Hinojo L, Ortiz H, Deus J, Menchon JM, and Cardoner N. 2013. Structural covariance of the neostriatum with regional gray matter volumes. Brain Structure and Function 218:697-709. 10.1007/s00429-012-0422-5.

Tarantini S, Tran CHT, Gordon GR, Ungvari Z, and Csiszar A. 2017. Impaired neurovascular coupling in aging and Alzheimer's disease: Contribution of astrocyte dysfunction and endothelial impairment to cognitive decline. Experimental Gerontology 94:52-58. 
568 Vaishnavi SN, Vlassenko AG, Rundle MM, Snyder AZ, Mintun MA, and Raichle ME. 2010.

569 Regional aerobic glycolysis in the human brain. Proceedings of the National Academy of

$570 \quad$ Sciences of the United States of Amecia 107:17757-17762. 10.1073/pnas.1010459107.

571 van den Heuvel MP, and Hulshoff Pol HE. 2010. Exploring the brain network: a review on

572 resting-state fMRI functional connectivity. European Neuropsychopharmacology 20:519534. 10.1016/j.euroneuro.2010.03.008.

574

575

576

577

578

579

580

581

582

583

584

585

586

587

588 2017. Abnormalities in the structural covariance of emotion regulation networks in major depressive disorder. Journal of Psychiatric Research 84:237-242.

10.1016/j.jpsychires.2016.10.001.

Xu G, Rowley HA, Wu G, Alsop DC, Shankaranarayanan A, Dowling M, Christian BT, Oakes TR, and Johnson SC. 2010. Reliability and precision of pseudo-continuous arterial spin labeling perfusion MRI on 3.0 T and comparison with 15O-water PET in elderly subjects at risk for Alzheimer's disease. NMR in Biomedicine 23:286-293. 10.1002/nbm.1462.

Yasuo K, Masayuki S, Hideki H, Hirofumi K, Makoto N, Tao C, Koichiro K, Kouji M, and Masatoshi F. 2002. Cerebral blood flow and vasodilatory capacity in anemia secondary to chronic renal failure. Kidney International 61:564-569. 10.1046/j.15231755.2002.00142.x.

Yen TH, Lin JL, Lin-Tan DT, and Hsu KH. 2009. Cardiothoracic ratio, inflammation, malnutrition, and mortality in diabetes patients on maintenance hemodialysis. American Journal of the Medical Sciences 337:421-428. 10.1097/MAJ.0b013e31819bbec1. 
589 Zhang LJ, Wen J, Liang X, Qi R, Schoepf UJ, Wichmann JL, Milliken CM, Chen HJ, Kong X, 590 and Lu GM. 2016. Brain Default Mode Network Changes after Renal Transplantation: A 591 Diffusion-Tensor Imaging and Resting-State Functional MR Imaging Study. Radiology 278:485-495. 10.1148/radiol.2015150004.

Zhang LJ, Wen J, Ni L, Zhong J, Liang X, Zheng G, and Lu GM. 2013. Predominant gray matter volume loss in patients with end-stage renal disease: a voxel-based morphometry study. Metabolic Brain Disease 28:647-654. 10.1007/s11011-013-9438-7.

Zheng G, Wen J, Yu W, Li X, Zhang Z, Chen H, Kong X, Luo S, Jiang X, Liu Y, Zhang Z, Zhang LJ, and Lu GM. 2016. Anemia rather than hypertension contributes to cerebral hyperperfusion in young adults undergoing hemodialysis: A phase contrast MRI study. Scientific Reports 6:22346. 10.1038/srep22346. Bulletin 43:1363-1374. 10.1093/schbul/sbx051. 
Figure 1

Figure 1. Reduced global CBF-ALFF coupling in hemodialysis patients.

Hemodialysis patients showed a significantly reduced $(P=0.0011)$ CBF-ALFF coupling compared to healthy controls. Significant difference was observed between two groups on CBF-ALFF correlation coefficients across voxels. Error bars represent the standard error. Abbreviations: CBF, cerebral blood flow; ALFF, amplitude of low-frequency fluctuation; HC, healthy controls; HD, hemodialysis patients. 


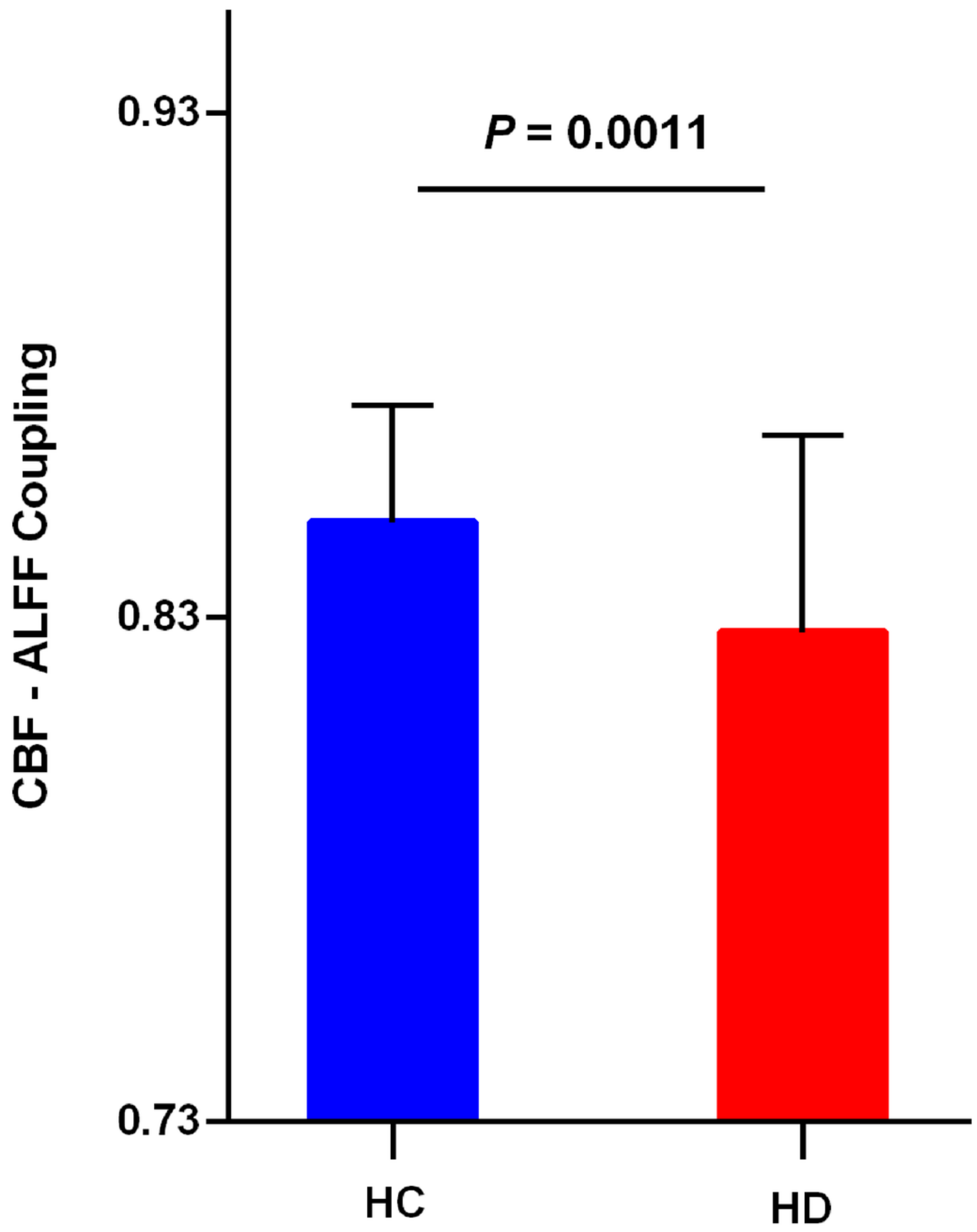




\section{Figure 2}

Figure 2. Spatial distribution maps of CBF/ALFF ratio in HC and HD.

Although somewhat differences were found in brain regions and magnitude, $\mathrm{HC}$ and HD groups exhibited relative consistent spatial distribution in these measures. Figure 2A presenting HC group and Figure 2B presenting HD group contain lateral (upper row) and medial view (bottom row), respectively. Abbreviations: CBF, cerebral blood flow; ALFF, amplitude of low-frequency fluctuation; HC, healthy controls; HD, hemodialysis patients; L, left; $R$, right.
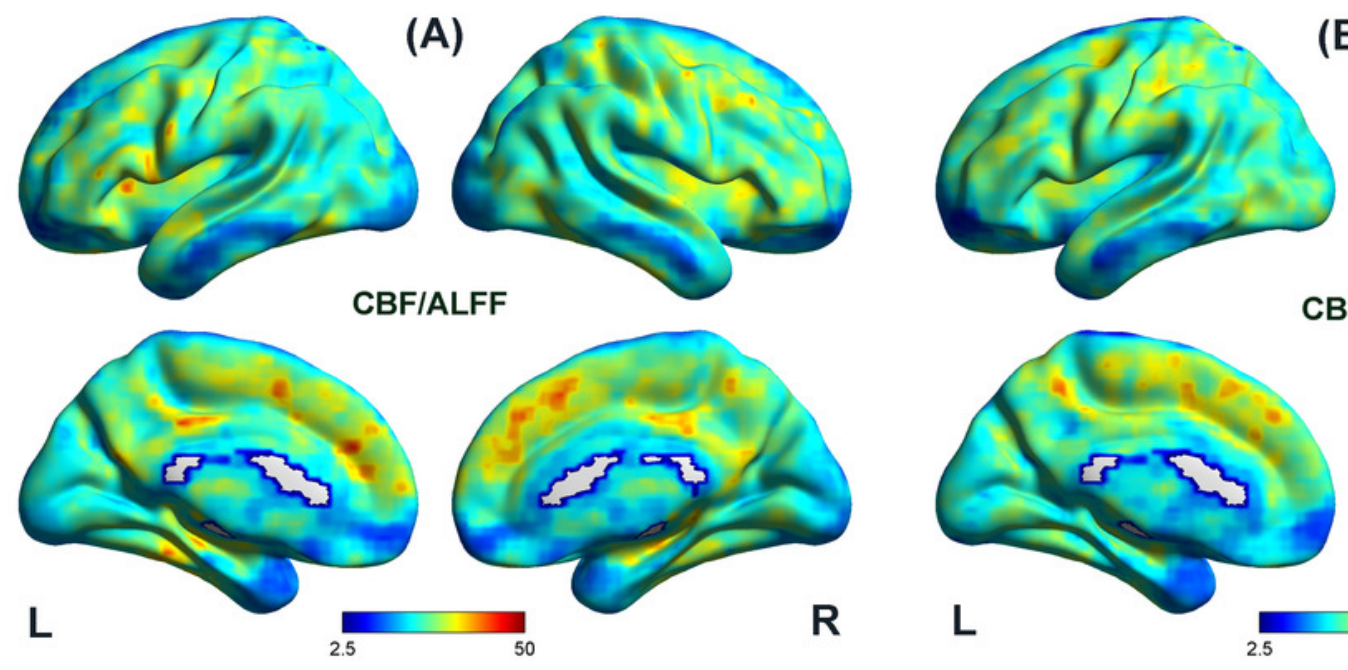

(B)
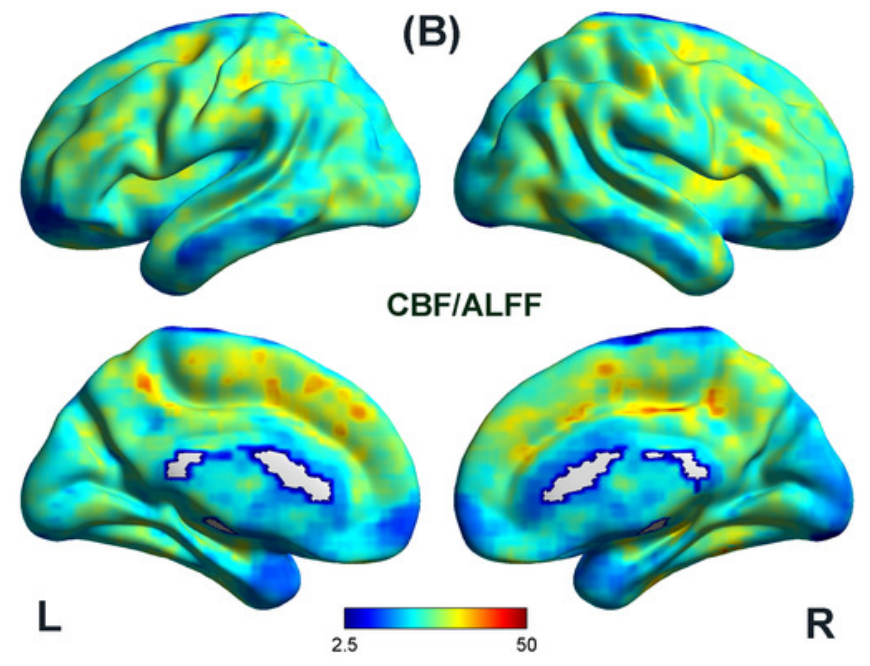


\section{Figure 3}

Figure 3. Group differences in CBF/ALFF ratio between hemodialysis patients and healthy controls.

Compared to healthy controls, hemodialysis patients showed decreased CBF/ALFF ratio in left

(Figure 3A-C) and right (Figure 3D-F) amygdala ( $P<0.05$, voxel-wise FDR correction).

Figure 3A and 3D, 3B and $3 \mathbf{E}$ as well as $\mathbf{3 C}$ and $\mathbf{3 F}$ represent coronal, axial and sagittal view, respectively. Abbreviations: CBF, cerebral blood flow; ALFF, amplitude of low-frequency fluctuation; R, right; FDR, false discovery rate.
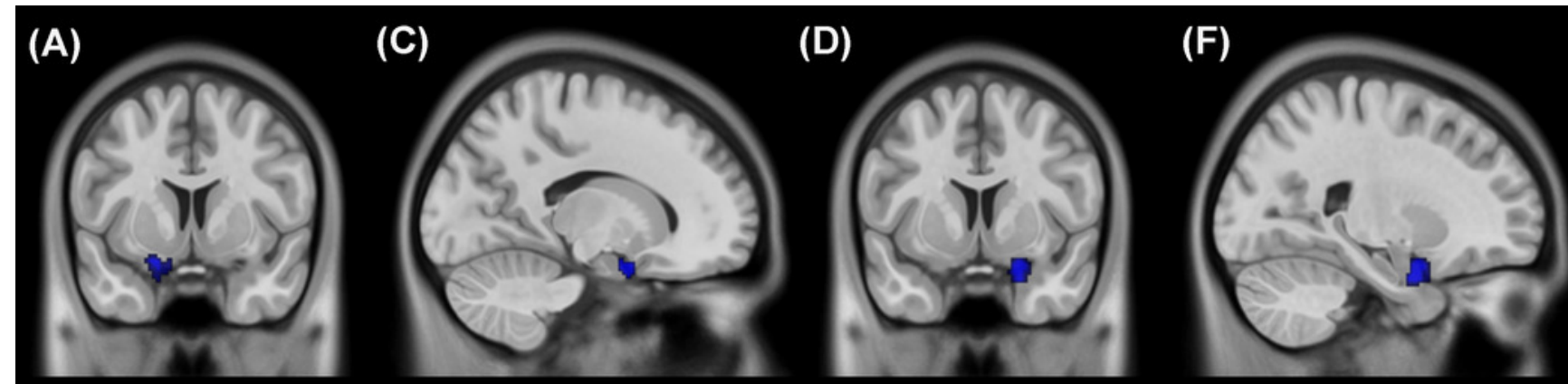

(B)

(E)

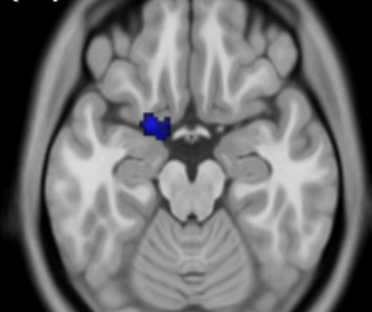

R

R 


\section{Figure 4}

Figure 4. Group differences in CBF between hemodialysis patients and healthy controls.

Compared with healthy controls, hemodialysis patients showed increased CBF in the thalamus bilaterally (Figure 4A-F) as well as decreased CBF in the right olfactory cortex and bilateral insula and anterior cingulated gyrus (Figure 4G-L) $(P<0.05$, voxel-wise FDR correction). Abbreviations: CBF, cerebral blood flow; R, right; FDR, false discovery rate.

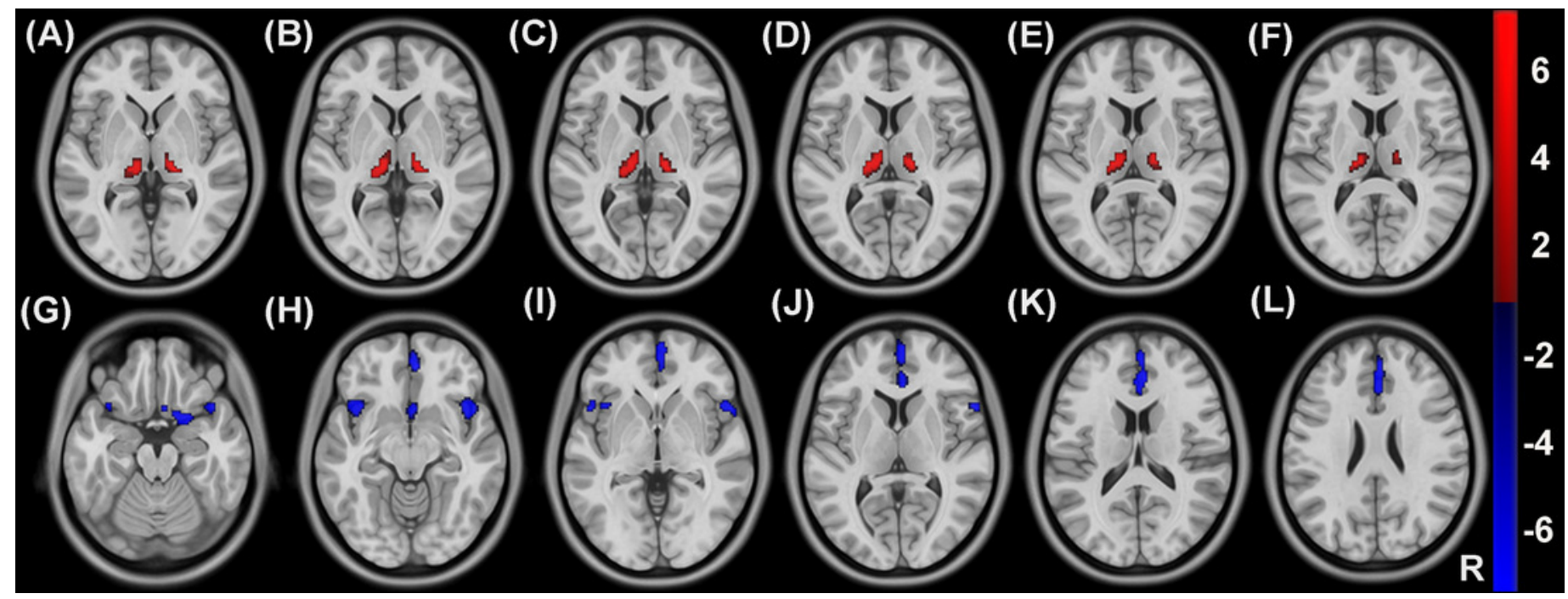




\section{Figure 5}

Figure 5. Group differences in ALFF between hemodialysis patients and healthy controls.

Compared with healthy controls, hemodialysis patients exhibited increased ALFF in the amygdala bilaterally (Figure 5A-F) as well as decreased ALFF in superior frontal gyrus and precuneus bilaterally (Figure 5G-L) ( $P<0.05$, voxel-wise FDR correction). Abbreviations: ALFF, amplitude of low-frequency fluctuation; R, right; FDR, false discovery rate.

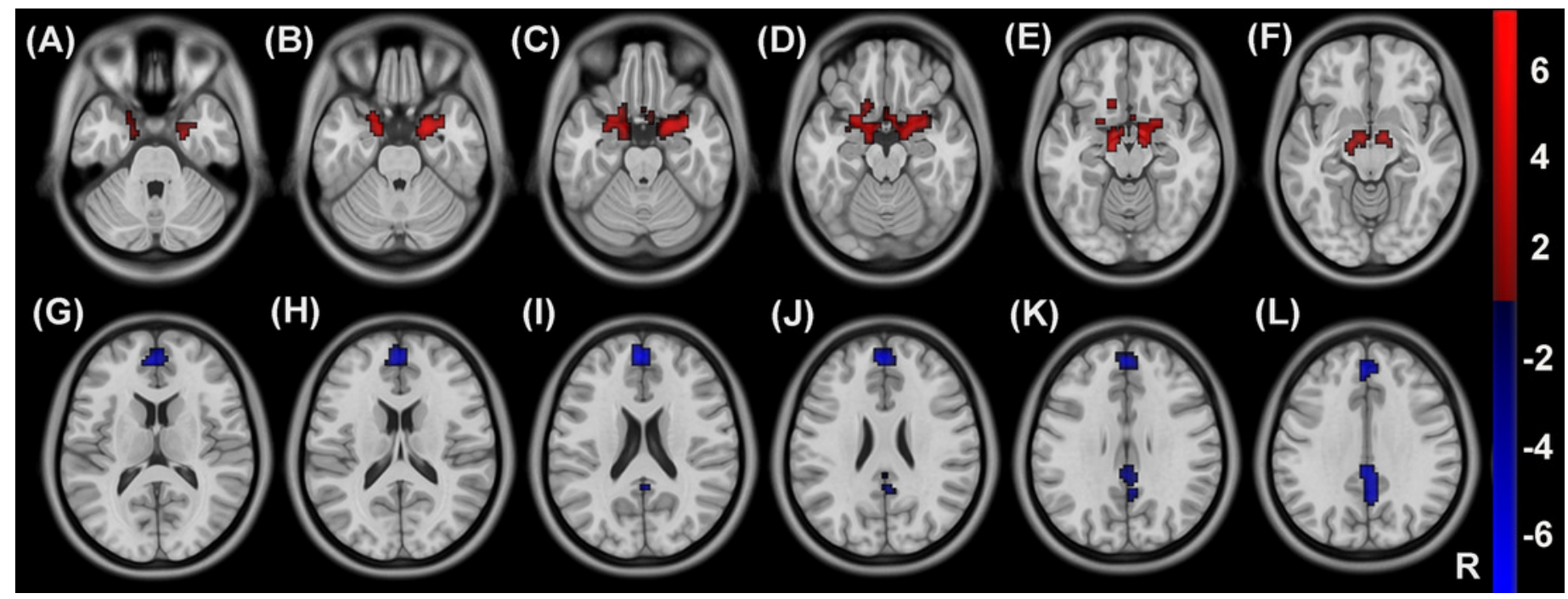




\section{Table $\mathbf{1}$ (on next page)}

Table 1. Demographic characteristics of HD and HC groups.

All values were expressed as mean \pm standard deviation or number of subjects. ${ }^{a}$ two-sample t-test. ${ }^{\mathrm{b}}$ Chi-square test. Abbreviation: HD, hemodialysis patients; $\mathrm{HC}$, healthy controls. 
Table 1. Demographic characteristics of HD and HC groups.

\begin{tabular}{|c|c|c|c|}
\hline Variables & $\mathrm{HD}(n=46)$ & $\mathrm{HC}(n=47)$ & $P$ Value \\
\hline Age (years) & $53.11 \pm 1.58$ & $55.57 \pm 0.86$ & $0.1263(t=1.543)^{\mathrm{a}}$ \\
\hline Gender (male/female) & $28 / 18$ & $22 / 25$ & $0.1739\left(\chi^{2}=1.849\right)^{\mathrm{b}}$ \\
\hline HD duration (HD times) & $484.58 \pm 186.31$ & - & \\
\hline Blood Pressure, $\mathbf{m m H g}$ & & - & \\
\hline Systolic Pressure & $145.91 \pm 20.54$ & - & \\
\hline Diastolic Pressure & $78.39 \pm 13.72$ & - & \\
\hline Blood tests & & - & \\
\hline Hemoglobin $(\mathrm{g} / \mathrm{l})$ & $114.30 \pm 12.15$ & - & \\
\hline Creatinine $(\mu \mathrm{mol} / \mathrm{l})$ & $935.01 \pm 222.41$ & - & \\
\hline Calcium (mmol/l) & $2.35 \pm 0.22$ & - & \\
\hline Phosphorus (mmol/1) & $1.46 \pm 0.55$ & - & \\
\hline Albumin $(\mathrm{g} / \mathrm{l})$ & $43.55 \pm 3.06$ & - & \\
\hline Blood Urea Nitrogen $(\mathrm{mmol} / \mathrm{l})$ & $20.55 \pm 4.95$ & - & \\
\hline Serum Ferritin $(\mathrm{ng} / \mathrm{ml})$ & $183.41 \pm 125.33$ & - & \\
\hline
\end{tabular}




\section{Table 2 (on next page)}

Table 2. Brain regions with significant group differences in CBF.

Multiple comparisons were corrected by a voxel-wise FDR method with a corrected threshold of $P<0.05$. Abbreviations: CBF, cerebral blood flow; FDR, false discovery rate; MNI, Montreal Neurological Institute; R, right; L, left; HD, hemodialysis patients; HC, healthy controls. 
Table 2. Brain regions with significant group differences in CBF.

\begin{tabular}{lccc}
\hline Regions & Cluster size (voxels) & Peak $t$ values & Coordinates in MNI (x, y, z) \\
\hline HD $<$ HC & 226 & -7.2060 & $18,8,-22$ \\
R Olfactory Cortex & 319 & -7.8958 & $44,14,-12$ \\
R Insula & 219 & -7.3120 & $-40,16,-12$ \\
L Insula & 833 & -7.4017 & $2,36,20$ \\
R and L Anterior Cingulate Gyrus & & & $18,-24,2$ \\
HD $>$ HC & 104 & 6.2191 & $-8,-18,8$ \\
R Thalamus & 187 & 6.0539 & \\
L Thalamus & & & \\
\hline
\end{tabular}




\section{Table 3 (on next page)}

Table 3. Brain regions with significant group differences in ALFF.

Multiple comparisons were corrected by a voxel-wise FDR method with a corrected threshold of $P<0.05$. Abbreviations: ALFF, amplitude of low-frequency fluctuation; FDR, false discovery rate; MNI, Montreal Neurological Institute; R, right; L, left; HD, hemodialysis patients; HC, healthy controls. 
Table 3. Brain regions with significant group differences in ALFF.

\begin{tabular}{lccc}
\hline Regions & Cluster size (voxels) & Peak $t$ values & Coordinates in MNI (x, y, z) \\
\hline HD $<$ HC & 308 & -5.5620 & $-3,51,24$ \\
R and L Superior Frontal Gyrus & 281 & -5.7403 & $9,-66,51$ \\
R and L Precuneus & & & \\
HD $>$ HC & 169 & 5.6808 & $21,3,-24$ \\
R Amygdala & 175 & 4.8168 & $-24,6,-21$ \\
L Amygdala & & &
\end{tabular}

1 